\title{
BMJ Open Feasibility and safety of an immersive virtual reality-based vestibular rehabilitation programme in people with multiple sclerosis experiencing vestibular impairment: a protocol for a pilot randomised controlled trial
}

\author{
Cristina García-Muñoz (i) ," María Jesús Casuso-Holgado (i) , \\ Juan Carlos Hernández-Rodríguez (1) ,' Elena Pinero-Pinto (D) , \\ Rocío Palomo-Carrión (D) ,3,4 María-Dolores Cortés-Vega (D) ${ }^{1}$
}

To cite: García-Muñoz C, Casuso-Holgado MJ, Hernández-Rodríguez JC, et al. Feasibility and safety of an immersive virtual realitybased vestibular rehabilitation programme in people with multiple sclerosis experiencing vestibular impairment: a protocol for a pilot randomised controlled trial. BMJ Open 2021;11:e051478. doi:10.1136/ bmjopen-2021-051478

\section{- Prepublication history and} additional supplemental material for this paper are available online. To view these files, please visit the journal online (http://dx.doi.org/10.1136/ bmjopen-2021-051478).

Received 19 March 2021 Accepted 22 October 2021

Check for updates

(C) Author(s) (or their employer(s)) 2021. Re-use permitted under CC BY-NC. No commercial re-use. See rights and permissions. Published by BMJ.

For numbered affiliations see end of article.

Correspondence to

Dr María Jesús Casuso-Holgado; mcasuso@us.es

\section{ABSTRACT}

Introduction Vestibular system damage in patients with multiple sclerosis (MS) may have a central and/or peripheral origin. Subsequent vestibular impairments may contribute to dizziness, balance disorders and fatigue in this population. Vestibular rehabilitation targeting vestibular impairments may improve these symptoms. Furthermore, as a successful tool in neurological rehabilitation, immersive virtual reality (VRi) could also be implemented within a vestibular rehabilitation intervention.

Methods and analysis This protocol describes a parallel-arm, pilot randomised controlled trial, with blinded assessments, in 30 patients with MS with vestibular impairment (Dizziness Handicap Inventory $\geq 16$ ). The experimental group will receive a VRi vestibular rehabilitation intervention based on the conventional Cawthorne-Cooksey protocol; the control group will perform the conventional protocol. The duration of the intervention in both groups will be 7 weeks (20 sessions, 3 sessions/week). The primary outcomes are the feasibility and safety of the vestibular VRi intervention in patients with MS. Secondary outcome measures are dizziness symptoms, balance performance, fatigue and quality of life. Quantitative assessment will be carried out at baseline (T0), immediately after intervention (T1), and after a followup period of 3 and 6 months (T2 and T3). Additionally, in order to further examine the feasibility of the intervention, a qualitative assessment will be performed at $\mathrm{T} 1$.

Ethics and dissemination The study was approved by the Andalusian Review Board and Ethics Committee, Virgen Macarena-Virgen del Rocio Hospitals (ID 2148-N-19, 25 March 2020). Informed consent will be collected from participants who wish to participate in the research. The results of this research will be disseminated by publication in peer-reviewed scientific journals.

Trial registration number NCT04497025.
Strengths and limitations of this study

- As the immersive virtual reality (VRi) intervention (experimental group) is developed and based on the Cawthorne-Cooksey conventional vestibular rehabilitation protocol (control group), it allows a homogeneous comparison between study groups.

- The VRi systems offer multisensory feedback, oriented tasks and repetitions of exercises in a ludic environment, thereby overcoming some of the limitations of the Cawthorne-Cooksey vestibular protocol.

Blinding of participants and therapists is not possible due to the type of intervention.

\section{INTRODUCTION}

Multiple sclerosis (MS) is a chronic autoimmune disease characterised by inflammation, demyelination of the central nervous system and axonal loss. ${ }^{12}$ Balance disorders, dizziness, and fatigue are among the most common and troublesome symptoms in MS, having repercussions on quality of life..$^{2-7}$ Fatigue is the most disabling manifestation in MS, of which impairments in central sensory integration may be an underlying cause. ${ }^{89}$ Furthermore, fatigue can be enhanced by vestibular symptoms such as vertigo, dizziness and imbalance. $^{1011}$

There is a myriad of vestibular system disorders, which could have a peripheral (inner ear, vestibular nerve) or central (brainstem and cerebellar) origin, or both. ${ }^{12-14}$ Balance problems, lack of coordination in cephalic movement with regard to the body, ocular disturbances and dizziness are symptoms related to vestibular disorders, as well as 
MS. ${ }^{11}{ }^{15-17}$ Postural deficits are associated with problems of the subjective visual vertical and dizziness during head movements, which are mediated by the vestibulo-ocular reflex (VOR). ${ }^{15}{ }^{18-20}$ Furthermore, impairments in the vestibulospinal reflex (VSR) can cause postural problems due to an inappropriate muscle response in imbalance situations. ${ }^{20-24}$ Central demyelination and/or peripheral disturbances can be possible aetiologies of vestibular impairments and their clinical manifestation in MS. ${ }^{25-28}$ Furthermore, the presence of vestibular impairments and their clinical manifestations may be affected by the progression of the disease.$^{1425-27}$ Specifically, patients with brainstem involvement, as identified using the Expanded Disability Status Scale (EDSS) could be showing signs of imbalance, vestibular disorders and greater disability. ${ }^{29} 30$

Vestibular rehabilitation consists of exercises that provide accurate spatial information of the head with regard to body position while stimulating VOR, VSR and somatosensory information. ${ }^{41-34}$ Based on mechanisms of substitution, adaptation and habituation, ${ }^{63}$ vestibular rehabilitation can be effective in addressing peripheral and central vestibular impairments. ${ }^{26} 3536$ Patients with MS therefore benefit from goals of vestibular rehabilitation, decreasing dizziness, improving ocular fixation and stability, and having better performance in daily living activities. ${ }^{33} 37-39$

Conventional vestibular rehabilitation consists of repetitive exercises and movements driven to improve physical or psychological impairments due to vestibular problems. ${ }^{40}$ Nowadays, Cawthorne-Cooksey vestibular training is considered the gold standard protocol within this framework. ${ }^{31} 41$ Although further research is needed, conventional vestibular training has been reported as superior to no intervention and at least as effective as exercise-based approach (Frenkel exercises and endurance training) for improving dizziness, balance and fatigue in any MS type. ${ }^{38}$ Currently, there is an exponential growth of studies that evaluate the effectiveness of virtual reality (VR) applied to vestibular rehabilitation in other diseases. ${ }^{42-50}$ The effectiveness of non-immersive VR for balance and gait training in patients with MS has already been proven. ${ }^{51}$ Moreover, a systematic review found that immersive VR (VRi) presents additional clinical benefits when compared with conventional vestibular training (performance and repetition of exercises in a motivational environment, oriented tasks, multisensory stimulation, extrinsic feedback and promotion of adherence).$^{52-57}$ The VR induces neuroplastic changes in neurological affection as MS. ${ }^{58}$ Within VRi, the modality that integrates physical activity in a virtual environment with mentioned advantages is exergame, that has proven to be effective for neurological diseases. ${ }^{59}$ Moreover, despite exercising through a VR system, it is perceived as less exhausting, ${ }^{61}$ while the subject is exposed to a large variety of environments boosting the vestibular mechanism of habituation. ${ }^{37} 62$ VRi allows the subject to complete immersion within the $360^{\circ}$ virtual environment, enhancing the feeling of presence. ${ }^{506364}$ To the best of

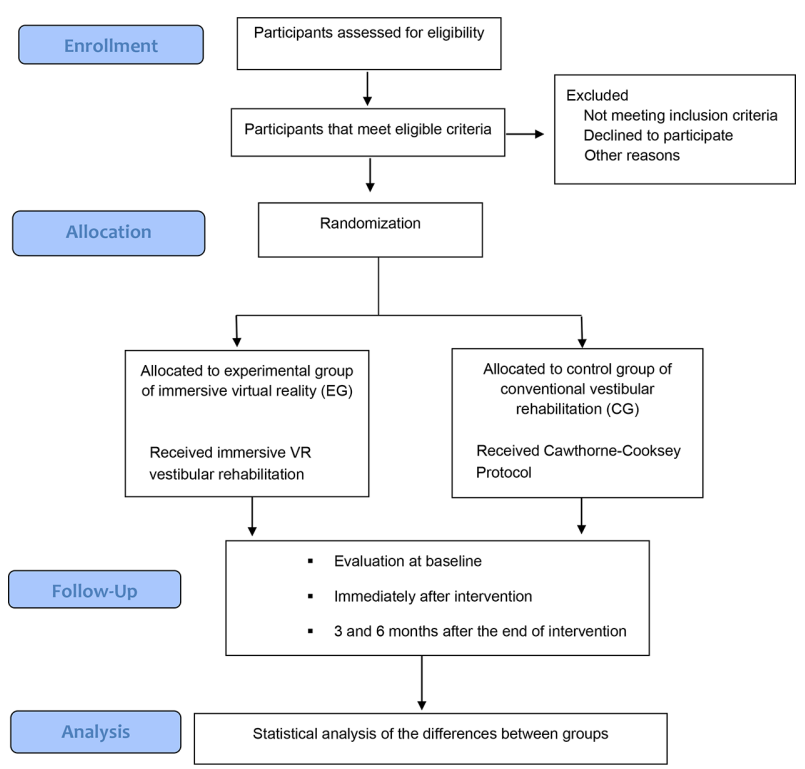

Figure 1 The Consolidated Standards of Reporting Trials flow diagram of the participants' recruitment and progress through the phases of the trial.

our knowledge, no previous research on VRi and vestibular rehabilitation in MS has been performed.

Therefore, the primary purpose of this study is to determine the feasibility and safety of a VRi-based vestibular rehabilitation programme in MS population. Second, we aim to preliminarily evaluate the preliminary effects of the vestibular VRi exercise protocol in comparison with conventional vestibular training for improvement in dizziness, balance, fatigue and quality of life in patients with MS.

\section{METHODS AND ANALYSIS \\ Study design}

This protocol describes a two-arm, parallel group, pilot randomised clinical trial (RCT), with blinded assessment. An initial evaluation of the study sample (T0) will be followed by an intervention period of 7 weeks for both the experimental group (EG) and control group (CG). A further three assessments will then be carried out immediately after intervention (T1) and after follow-up periods of 3 (T2) and 6 months (T3). The study design is illustrated in figure 1 .

This protocol meets the Standard Protocol Items: Recommendations for Interventional Trials. ${ }^{65}$ This RCT will also be developed following instructions from the Consolidated Standards of Reporting Trials. ${ }^{66}$

\section{Study setting}

The trial will be conducted at the Physical Therapy Department of the University of Seville (Spain). The Virgen Macarena Hospital will be the main healthcare institution involved in this study. The inclusion of other healthcare centres in the area is expected. 


\section{Participants and recruitment}

Recruitment of participants is expected to start in September 2021 and end in September 2022. All subjects who potentially meet the eligibility criteria will be contacted to participate in the study. Those who decide to participate and meet the eligibility criteria will be asked for written informed consent (please see online supplemental material for informed consent form).

\section{Inclusion criteria}

- Both male and female subjects aged 18-65 years.

- Clinically diagnosed with any type of MS in accordance with the revised McDonald criteria. This will be assessed based on clinical history by a medical team.

- Walking ability according to the EDSS score (EDSS $\leq 6)$. This will be assessed based on clinical history by a medical team.

- Brainstem or cerebellar involvement with $\geq 2$ points in the second functional system of the EDSS. ${ }^{67}$ This will be evaluated based on clinical history by a medical team.

- Objective presence of dizziness symptoms (Dizziness Handicap Inventory (DHI) $\geq 16$ ). This will be assessed after informed consent acceptance by an expert vestibular physical therapist.

- Presence of fatigue (Modified Fatigue Impact Scale (MFIS) $\geq 38)^{68}$ or balance problems (Berg Balance Scale $(\mathrm{BBS}) \leq 47) .{ }^{69}$ This will be evaluated after the acceptance of participation in the study by an expert vestibular physical therapist.

\section{Exclusion criteria}

- Partial or complete blindness.

- Cognitive impairment (Mini-Mental State Examination score $\leq 24)$.

- Another neurological disorder contributing to balance impairment.

- Disease relapse within the last 3 months (transitory exacerbation of the disease by the appearance of neurological clinical manifestations: imbalance, dizziness and more). ${ }^{27} 7071$

- Changes in MS pharmacotherapy within the last 3 months.

- History of vestibular rehabilitation within the last 6 months.

- Acute cardiovascular or respiratory illnesses.

- Contraindications to VRi use (epilepsy, spatiotemporal disorientation and cognitive impairment).

- Any other contraindications to physical activity.

Exclusion criteria will be assessed based on clinical history by a medical team.

\section{Randomisation, concealment allocation and blinding}

Participants will be randomly allocated to one of the two intervention groups by an independent researcher, using 1:1 distribution ratio and a computer-generated random sequence. The independent researcher will oversee the randomisation process and place the allocation of participants in sealed and concealed envelopes. This researcher will inform participants of their random allocation and will provide them the informed consent forms. An expert physical therapist in vestibular rehabilitation will perform the intervention. The assessor will remain blinded to the participants' groups.

\section{Patient and public involvement}

No patients or members of the public are involved in designing the trial, but a number of public organisations will be contacted for patient recruitment (for example, Hospital Virgen Macarena, Ilustre Colegio Profesional de Fisioterapeutas de Andalucía). However, based on their experiences in this pilot study, participants will play a significant role in remodelling the intervention and tailor it to the specific needs of patients with MS. For this purpose, a qualitative evaluation performed through a semistructured interview process for each participant will be included. This triangulation method will help us to interpret the study findings. ${ }^{72}$

Once the study is completed, participants will be informed about it by email in a comprehensible writing style. Furthermore, the researchers will host meetings in each public organisation engaged in recruitment.

\section{Interventions}

Conventional vestibular rehabilitation protocol (control group)

The control group (CG) will perform the conventional vestibular rehabilitation Cawthorne-Cooksey protocol exercises. $^{31}$ These exercises aim to restore balance affected by vestibular dysfunction and train the vestibular system. Subsequently, this may improve vestibular compensation through a mechanism of neuroplasticity, known as adaptation, habituation and substitution. ${ }^{37} 6273$ The primary goal of these mechanisms is to adapt the VOR and VSR, habituate and substitute head movements that provoke vestibular and balance symptoms, and train dynamic balance.

As shown in table 1, exercises are divided into three blocks, which will be performed slowly at first and then progressively faster. Participants allocated to the CG will receive this conventional protocol three times per week for 7 weeks. Each session will last for $50 \mathrm{~min}$, and the rest time will be for at least $5 \mathrm{~min}$. A total of 10 initial sessions and 10 advanced sessions will be carried out. Based on previous studies, during the initial phase, exercises of the first and second blocks will be carried out by 10 slow repetitions and 10 fast repetitions. ${ }^{74} 75$ The third block exercises will be repeated five times slowly and then five times more quickly. The complete intervention time for each block is $15 \mathrm{~min}$ (table 1). Once participants have exceeded the first 10 sessions, they will begin with more complex exercises. To develop these advanced vestibular exercises for both groups, the principles and keys of Cooksey, ${ }^{31}$ Han $e t a l^{37}$ and Whitney and Sparto ${ }^{62}$ were assumed. The advanced phases of the intervention for participants in the CG are described in table 2. This intervention matches the EG, with the only 
Table 1 Description of initial phase of vestibular intervention in both groups of study based on convectional protocol of Cawthorne-Cooksey exercises

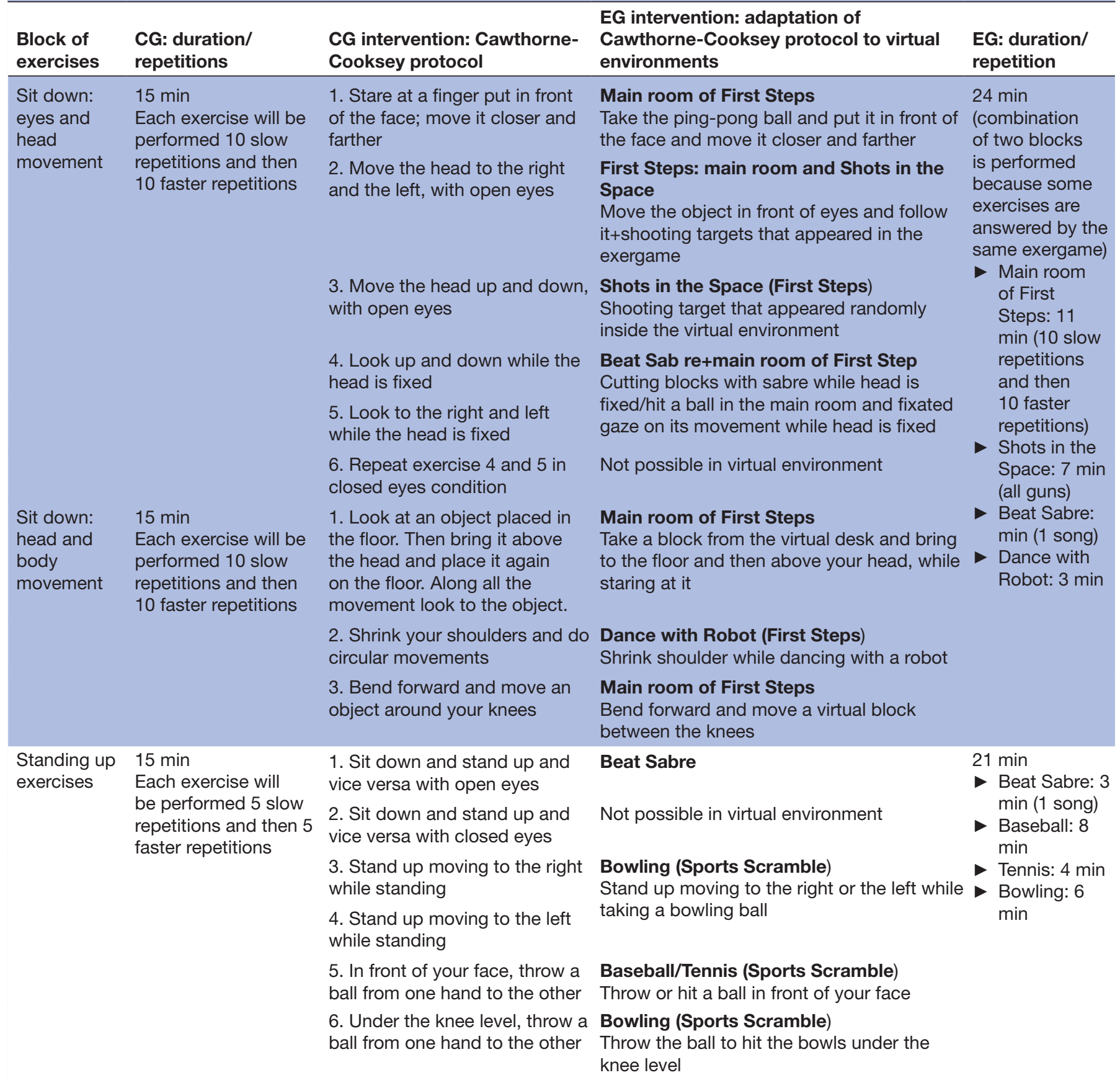

CG, control group; EG, experimental group.

difference being that exercises are not performed in an immersive virtual environment. The exercise parameters in the advanced sessions are the amplitude of the support base, alternative single leg support, tandem position, unstable surface and walking with head movements. To avoid the appearance of vestibular symptoms during exercises, these parameters will be carried out in the specific order mentioned above. These parameters provide proprioceptive disturbances and encourage vestibular training through substitution of neural mechanisms. ${ }^{3762}$ Other parameters that train habituation and adaptation mechanisms include the increasing speed of head movement or its range of motion. ${ }^{3762}$ All parameters can be adapted to patient characteristics and progress with each session (for example, modifying the base of support from higher to lower amplitude on the firm and unstable surface).

The vestibular programme will be conducted by an experienced vestibular rehabilitation physical therapist, who will provide verbal indications and stay near the 
Table 2 Description of advanced phase of vestibular exercises for both groups

\begin{tabular}{ll}
$\begin{array}{l}\text { Exercises for both } \\
\text { groups }\end{array}$ & $\begin{array}{l}\text { CG: duration and } \\
\text { frequency }\end{array}$ \\
\hline
\end{tabular}

\begin{tabular}{|c|c|c|}
\hline $\begin{array}{l}\text { 1. Changing from sitting } \\
\text { to standing and vice } \\
\text { versa }\end{array}$ & 10 repetitions & $\begin{array}{l}\text { From a situation of } \\
\text { sitting in a chair, } \\
\text { stand up and throw } \\
\text { a ball }\end{array}$ \\
\hline
\end{tabular}

\section{Move and throw an} object from one hand to the other while standing with feet together.

10 repetitions moving the object 10 repetitions throwing the object

\section{Move a ball at eye} level and then throw it from one hand to the other

\section{EG}

\section{Main room of First Steps}

Take a block from virtual desk and when the subject stands up, throw it a virtual sign situated inside the virtual environment

\section{Main room of First Steps}

Move a virtual block at eye level

Take a virtual block and throw it from one hand to the other
EG: duration and frequency

10 repetitions

Staring all the time to the object

$\begin{array}{lll}\begin{array}{l}\text { 3. } 360^{\circ} \text { turn } \\ \text { right/left }\end{array} & \begin{array}{l}10 \text { repetitions to the } \\ \text { a ball to a target }\end{array} \\ \begin{array}{l}\text { 4. Moving the head with } 360^{\circ} \text { and throw } \\ \text { narrow base of support }\end{array} & \begin{array}{l}15 \text { repetitions (eg, } \\ \text { the right) }\end{array} & \begin{array}{l}\text { Move head to right } \\ \text { and left with feet } \\ \text { together }\end{array}\end{array}$

5. Stare at an object put 10 slow repetitions
in front of the face; move 10 fast repetitions
it closer and farther
while standing on a foam
surface

\section{Fast side head} movements while standing on a foam surface

7. Move an object to the 10 repetitions
floor and bring it above
your head while standing
on a foam surface

15 repetitions
15 repetitions

8. Head movements while alternative single leg support
Stare at a small ball and move it closer or farther to your face

\section{Main room of First Steps}

Take a virtual block, turn $360^{\circ}$ and throw right/left it to a located target in the environment

\section{Main room of First Steps}

In standing position with narrow base of support, hit a ball and follow with the head its movements

\section{Main room of First Steps}

Take the ping-pong ball and put it in front of the face and move it closer and farther

Throwing a ball to the Main room of First Steps right and left while standing on a foam surface. Follow the ball with the head

Taking a ball and
make the exercise

Take the ping-pong racket and hit blocks to one side and another following them with the head make the exercise

\section{Main room of First Steps}

Taking a virtual block from the desk, perform the exercise

Look to the right and the left while you maintain a monopodal balance

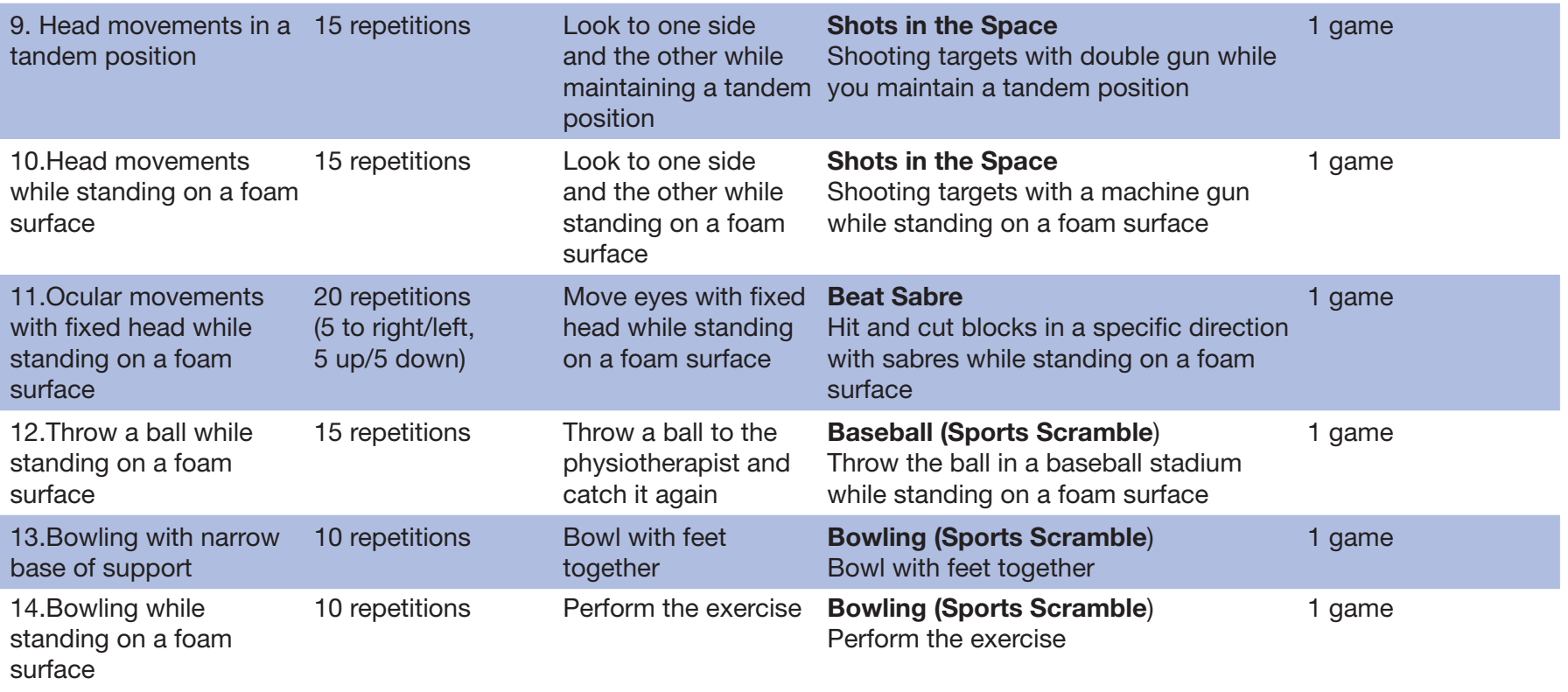

Continued
10 repetitions moving the object

10 repetitions

throwing the object 


\begin{tabular}{|c|c|c|c|c|}
\hline $\begin{array}{l}\text { Exercises for both } \\
\text { groups }\end{array}$ & $\begin{array}{l}\text { CG: duration and } \\
\text { frequency }\end{array}$ & CG & EG & $\begin{array}{l}\text { EG: duration and } \\
\text { frequency }\end{array}$ \\
\hline $\begin{array}{l}\text { 15. Head movements } \\
\text { while walking through a } \\
\text { corridor }\end{array}$ & 20 repetitions & $\begin{array}{l}\text { Walk down a corridor } \\
\text { while moving head }\end{array}$ & $\begin{array}{l}\text { Bowling (Sports Scramble) } \\
\text { Walk down a bowling alley, while moving } \\
\text { head side to side and then throw the } \\
\text { bowling ball }\end{array}$ & 2 games \\
\hline
\end{tabular}

CG, control group; EG, experimental group.

participants to lend them confidence and decrease the risk of falling during the session.

\section{VRi intervention (EG)}

Participants assigned to the EG will receive VRi vestibular rehabilitation through the head-mounted display (HMD) Oculus Quest (Facebook Technologies). VRi allows complete immersion in a $360^{\circ}$ virtual environment and enables interaction. Immersive virtual rehabilitation can only be achieved with the use of a VR headset or HMD. In this protocol, the new generation Oculus Quest equipment has been selected, which has some added advantages compared with other similar HMDs. These advantages include the absence of movement sensors or laptop installations, wireless option, portability and a reduced risk of suffering from cybersickness syndrome, owing to the high resolution and accurate movement capture. ${ }^{767}$

To achieve homogeneous interventions between the two groups, the VRi intervention has been designed based on the gold standard Cawthorne-Cooksey vestibular protocol. Subjects in this group will receive the same number of sessions and duration as the CG. Similar to the CG, the first 10 sessions of the VRi treatment will be carried out in the sitting down position (eyes and head movement/head and body movement) and the last one as standing up exercises. The number of repetitions and adaptation of VRi equated to the conventional protocol for immersive virtual environments during the initial phase is described in table 1 . In the initial phase, the advanced phase exercises will be the same in both groups, with the main difference being the interaction with the immersive virtual environment. The advanced phases of vestibular rehabilitation and the VRi-adapted exercises are shown in table 2. The exercise parameters described in the CG will be applied in the EG as well. In addition, to prevent falls over interaction with virtual environments, participants will be monitored and supervised by an expert physical therapist.

First Steps, Beat Sabre demo and Sports Scramble demo games will be displayed using the Oculus Quest HMD to apply the vestibular protocol. These games reflect a first-person exergame environment in which subject actions are recreated virtually. Furthermore, all selected games are commercially available and have free access in the Oculus app to anyone who owns an HMD device. First Steps is the onset game of Oculus, in which one learns to use the VRi device in a playable way. This game consists of the main room where the subject can interact with virtual objects as virtual blocks, ping-pong racket and ball, hanging ball and more. First Steps also contains two additional virtual environments. The first is a shooter game called Shots in the Space, which aims to reach the highest score while shooting random targets at a space station. This shooter offers three options: a single gun, a double gun or a machine gun, which will be included in exercises. The second is Dance with Robot, in which one dances and interacts with a robot. Beat Sabre is a rhythm music game in which blocks are slashed in a specific direction with a red (left hand) and blue (right hand) sabre, while trying to avoid some obstacles. Sports Scramble consists of three sports games: baseball, tennis and bowling, in which one must defeat their opponent while balls, rackets or your baseball bat is randomly changing into a giraffe, a cheese and so on. The virtual scenarios are shown in figure 2.

\section{Outcomes and measurements}

The primary outcomes will include the feasibility and safety of the experimental VRi vestibular protocol. The feasibility of the study will be assessed using recruitment, adherence, retention rates and usability of the VRi device. In addition to this quantitative assessment, semistructured interviews will be conducted with the VRi intervention

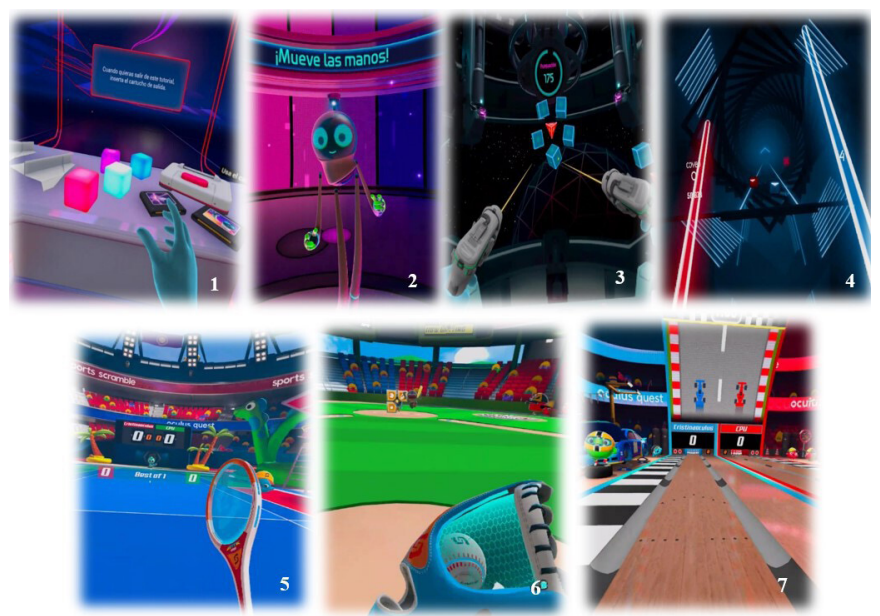

Figure 2 Virtual environments of exergames from the VRi vestibular rehabilitation, Oculus Quest, Facebook. (1) Main room of First Steps; (2) Dance with Robot; (3) Shots in the Space; (4) Beat Sabre; (5) Tennis (Sports Scramble); (6) Baseball (Sports Scramble); (7) Bowling (Sports Scramble). VRi, immersive virtual reality. 
Table 3 Primary outcomes' predefined thresholds

\begin{tabular}{|c|c|c|}
\hline Feasibility measurements & Measure & Predefined thresholds \\
\hline Recruitment/participation rate ${ }^{84}$ & $\begin{array}{l}\text { Proportion of potential participants who agree to } \\
\text { complete screening and consent to participate }\end{array}$ & $\geq 65 \%$ \\
\hline Retention rate 84 & $\begin{array}{l}\text { Proportion of participants with complete study data at } \\
\text { 3-month and 6-month follow-up }\end{array}$ & $\geq 75 \%$ \\
\hline \multicolumn{3}{|l|}{ Safety measurements } \\
\hline Cybersickness $^{88}$ & SSQ & $\leq 15$ points \\
\hline Fatigue to exercise ${ }^{89}$ & ROF & $\leq 4$ points \\
\hline Adverse events & Session's registry & No between-group differences \\
\hline
\end{tabular}

ROF, Rating of Fatigue; SSQ, Simulator Sickness Questionnaire; SUS, System Usability Scale.

participants. The interview will be carried out by the therapist in charge of the intervention. This qualitative strategy is expected to allow a deeper understanding of the participants' experiences. Safety will be examined by the appearance of cybersickness and fatigue to exercise along the VR treatment and a registry of falls and other adverse events. Predefined thresholds for considering the feasibility and safety of the VRi intervention are described in table $3 .^{78-83}$

Secondary outcomes include changes in dizziness, balance, fatigue and quality of life after a VRi vestibular protocol compared with conventional vestibular rehabilitation.

\section{Usability of the VR system}

In combination with participation, retention and adherence to treatment rates, feasibility will be evaluated using the System Usability Scale (SUS). The SUS is a 10-item questionnaire in which participants consider their perception of the VR device usability using a 5-point Likert scale, where 0 means 'strongly disagree' and 5 means 'strongly agree'. The overall score ranges from 0 to 100 , which is obtained by multiplying the sum of every item by 2.5 . A higher score indicates higher usability. ${ }^{80}{ }^{81}$ To maintain the blindness of the assessor, this measurement will be performed by the physiotherapist who conducted the intervention.

\section{Cybersickness syndrome}

To assess the safety of the intervention along with the fall and adverse events registry, the appearance of cybersickness will be evaluated using the Simulator Sickness Questionnaire (SSQ). The SSQ is implemented to measure the appearance of sickness due to a virtual environment. The SSQ is a 16-item questionnaire divided into three categories: nausea, oculomotor and disorientation. ${ }^{845}$ Scores ranging between 10 and 15 indicate significant symptoms, and those above 20 indicate a simulator problem. ${ }^{82}$ This scale will be provided by the physical therapist during each session.

\section{Rating of Fatigue Scale}

To examine safety along with the performance of the sessions, the appearance of fatigue related to exercise will be evaluated through Rating of Fatigue (ROF) ${ }^{83}$ This scale is a visual analogue rating scale ranging from 0 (nonfatigue) to 10 (totally fatigued/exhausted). The main aim of this scale is to assess fatigue in myriad contexts while exercising or during daily living activities. The ROF will be presented to the participants in each session.

\section{Dizziness}

Dizziness symptoms will be assessed using the DHI. This self-assessment questionnaire consists of 25 items divided into the following subscales: physical, emotional and functional. The physical and emotional subscales range from 0 to 36 points, and the functional subscale ranges from 0 to 28 points. The total score is 100 , which relates to the highest level of disability and handicap. ${ }^{86-88}$ This instrument is reliable and valid for the study population. ${ }^{89} 90$ The minimal clinical importance difference (MCID) has been established at 18 points in patients with vestibular disorders. ${ }^{88}$

\section{Balance}

Static balance will be evaluated using the Biodex Balance System. The aforementioned system allows the registration of the location of the centre of pressure (CoP)..$^{91-93}$ Biodex has been proven to be a valid instrument for evaluating stability and postural control in subjects with MS. ${ }^{9495}$ Moreover, Biodex can compute the following variables in relation to the CoP:

- Length $(\mathrm{mm})$, the CoP trajectory throughout the platform surface.

- Anteroposterior and mediolateral sway; these measure CoP deviation along each axis $(\mathrm{mm})$.

- Velocity $(\mathrm{mm} / \mathrm{s})$ of CoP oscillation through the anteroposterior axis and mediolaterally.

Each variable will be assessed in open or closed eyes condition and on a firm or foam surface, respectively. 
The BBS will be used to measure dynamic balance. The BBS consists of 14 items, each ranging from 0 (cannot perform) to 4 (normal performance), where higher values indicate better dynamic balance. ${ }^{96} 97$ This assesses the skills of sitting, standing, leaning, turning and standing on a monopodal support. The BBS has proven to be reliable and valid for the study population. ${ }^{89} 90$ The MCID for BBS has been set at 3 points for people with MS by Gervasoni et al. ${ }^{98}$

\section{Fatigue}

The MFIS is a self-reported questionnaire that evaluates the perceived impact of fatigue in patients with MS. This scale is composed of 21 items which assess the fatigue impact in three different domains. The global scale is divided into 9, 10 and 2 items that belong to the physical, cognitive and psychosocial domains, respectively. The total score is 84 , with higher scores indicating a higher impact of fatigue. ${ }^{99} 100$ This scale is reliable and valid for measuring the impact of fatigue in patients with MS. ${ }^{101} 102$ The MCID for MFIS has been established at $19.23 \%$ by Rietberg et al ${ }^{103}$ and 4 points by Roony et al. ${ }^{104}$

\section{Quality of life}

To assess the changes perceived by participants in their quality of life, the reliable and valid Multiple Sclerosis Quality of Life Scale 54 will be used. ${ }^{105}$ This is a 54 -item questionnaire distributed into 12 multi-item scales. The overall score ranges from 0 to 100 . Higher values indicate a better quality of life. ${ }^{106}$
Data will be collected by a blinded physical therapist who is an expert in neurological and vestibular rehabilitation. The blind evaluation will be performed at several points in the study: before the intervention, at the end of the intervention, and at 3 and 6 months post-intervention (table 4).

\section{Sample size calculation}

A major reason for conducting a pilot study is to determine the initial data to perform a sample size calculation for a larger trial. ${ }^{76}$ For this reason, the formal sample size will not be carried out. However, following the recommendations of good practice for the design and analysis of feasibility and pilot studies in preparation for RCT, ${ }^{7677}$ we aimed to recruit at least 30 subjects ( 15 per group).

\section{Statistical analysis}

To assess the feasibility and safety of the experimental VRi intervention, a descriptive data analysis will be implemented, taking into consideration the predefined thresholds for the primary outcomes (table 3). Participants' flow will be analysed to report the proportion of subjects who are eligible, consenting, adhering to intervention, and have retention rates at 3 and 6 months. These data will help to identify possible modifications in the definitive trial design when VRi is found feasible and safe.

The normal distribution of the variables will be assessed using the Shapiro-Wilk test. For normal distribution, data will be reported as mean \pm SD or as percentages. Similarly, for non-normal distribution, median, minimum and

\begin{tabular}{|c|c|c|c|c|c|c|c|}
\hline $\begin{array}{l}\text { Data and } \\
\text { outcomes of } \\
\text { study }\end{array}$ & Assessment details & $\begin{array}{l}\text { Screening } \\
\text { and } \\
\text { recruitment }\end{array}$ & $\begin{array}{l}\text { Baseline } \\
\text { (T0) }\end{array}$ & $\begin{array}{l}\text { During } \\
\text { intervention }\end{array}$ & $\begin{array}{l}\text { After } \\
\text { intervention } \\
(\mathrm{T} 1)\end{array}$ & $\begin{array}{l}\text { Follow-up at } \\
3 \text { months } \\
\text { (T2) }\end{array}$ & $\begin{array}{l}\text { Follow-up at } 6 \\
\text { months } \\
\text { (T3) }\end{array}$ \\
\hline $\begin{array}{l}\text { Eligibility } \\
\text { assessment }\end{array}$ & & $x$ & & & & & \\
\hline $\begin{array}{l}\text { Demographic } \\
\text { variables }\end{array}$ & & $x$ & & & & & \\
\hline Safety & $\begin{array}{l}\text { Cybersickness: SSQ } \\
\text { Fatigue to exercise: ROF } \\
\text { Falls/adverse events registry }\end{array}$ & & & $x$ & & & \\
\hline Dynamic balance & BBS & & $x$ & & $x$ & $x$ & $x$ \\
\hline Fatigue & MFIS & & $x$ & & $x$ & $x$ & $x$ \\
\hline Quality of life & MSQoL-54 & & $x$ & & $x$ & $x$ & $x$ \\
\hline
\end{tabular}

BBS, Berg Balance Scale; DHI, Dizziness Handicap inventory; MFIS, Modified Fatigue Impact Scale; MSQoL-54, Multiple Sclerosis Quality of Life Scale 54; ROF, Rating of Fatigue; SSQ, Simulator Sickness Questionnaire; SUS, System Usability Scale. 
maximum values, and IQRs will be reported. Baseline differences between groups will be analysed using the $\mathrm{X}^{2}$ test for categorical variables and the t-test or MannWhitney $\mathrm{U}$ test for continuous variables. This will help identify possible covariates.

Linear mixed models will be used to test group, time and group-by-time interaction effects for all secondary variables on an intention-to-treat basis. The analyses will be first unadjusted for any baseline characteristics and later adjusted for possible identified covariates (for example, gender or EDSS scores).

Cohen's criteria will be followed to value the effect sizes of the studied variables, though due to the pilot nature of the study, all the effect analyses must be considered exploratory only. Nonetheless, these data will help in sample size calculations for a definitive RCT. For all tests, $\mathrm{p}<0.05$ will be considered statistically significant. Graphical and numerical analysis of the data will be conducted using SPSS (V.25.0; IBM Corp) and GraphPad PRISM (GraphPad, San Diego, California, USA).

\section{Data management and monitoring}

The study will not have an independent data monitoring committee because the main decisions will be agreed between the members of the research team. All data will be codified and recorded in an encrypted database by a number (instead of the subjects' name, for example) known only by the researcher team. The data will not be disclosed to third parties without participant consent.

Falls or any other adverse events derived during the intervention will be recorded by the therapists in a registry. These events will be communicated to the principal investigator of the study.

\section{ETHICS AND DISSEMINATION}

The study was approved by the Andalusian Review Board and Ethics Committee Virgen Macarena-Virgen del Rocio Hospitals (ID 2148-N-19, 25 March 2020). All participants will undergo and provide informed consent before data compilation. The investigators will disseminate the study results through literature in peer-reviewed scientific journals.

\section{DISCUSSION}

The current protocol for this pilot RCT aims to assess the feasibility and safety of vestibular rehabilitation in patients with MS through a VRi intervention compared with the conventional approach. Likewise, we will evaluate the changes that occurred in dizziness, postural control, fatigue and quality of life for both study groups after the vestibular intervention.

\section{Technical progress of VRi}

The Cawthorne-Cooksey vestibular protocol presents some limitations like the absence of feedback, no changes in the surface of work, and lack of cognitive and task-oriented training; thus, vestibular training is based on repetitive exercises performed without a functional objective or variability in the environment. ${ }^{41}{ }^{44}$ Due to the intrinsic advantages of VRi and the multimodal design ${ }^{107}$ of the protocol, the limitations of the Cawthorne-Cooksey training are expected to be overcome by providing extrinsic feedback (game score and multisensorial stimulation) during exercise execution, possibility of adding changes in surface and base of support during the performance, cognitive and task-oriented training (exergames), and avoiding humdrum exercise repetitions because of the motivational and enjoyable environment. ${ }^{52} 107$

Owing to VRi tracking (gyroscopes, accelerometers and magnetometers) and software systems that record head and corporal movements in $6 \mathrm{df}$, it is possible to perform exercises in different postural circumstances, similar to our experimental protocol (sitting down, standing, single leg support, tandem and standing on foam surface), ensuring virtual environment verticality. ${ }^{77} 108$ Furthermore, the command centre of movements and multisensory stimulation are primarily found at the cephalic level in HMD, making VRi a suitable device for vestibular rehabilitation. ${ }^{84} 109-111$ Moreover, current VRi devices are affordable, own high-resolution graphics, and have higher frames per second, less delay and latency, and accurate software and hardware. ${ }^{112} 113$ These enhance the sense of presence and immersion of the subject and reduce the possible appearance of cybersickness, as confirmed by Weech et l. $^{114}$

\section{Clinical applicability of VRi vestibular rehabilitation}

The Cawthorne-Cooksey intervention, on which our VRi protocol is based, has been demonstrated to be effective in several populations, such as elderly people, ${ }^{107}$ people with vertebrobasilar insufficiency ${ }^{115}$ and those with benign paroxysmal positional vertigo. ${ }^{116}$ Thus, arguably, vestibular VRi intervention based on this gold standard could be effective in the mentioned populations, including patients with MS. Promising previous studies have reported the effectiveness of VRi in vestibular rehabilitation for unilateral vestibular hypofunction, ${ }^{46}{ }^{48}$ Meniere's disease ${ }^{4344}$ and traumatic brain injury. ${ }^{117}$ Moreover, a recent systematic review by Soltani and Andrade ${ }^{118}$ supports HMD as a feasible and safe intervention to improve balance in older adults; because of this, we hypothesise that VRi vestibular intervention will be safe and feasible in MS population. ${ }^{19-122}$

Finally, telerehabilitation strategies combined with VR have been poorly studied in the MS population. ${ }^{123} \mathrm{~A}$ recent study with 10 participants with MS showed satisfactory results in balance and gait, but not for fatigue, after a telerehabilitation intervention based on Nintendo Wii exergames. ${ }^{124}$ With regard to our protocol, because Oculus Quest is wireless and portable, exercises can be performed at the laboratory, in public, in private clinics and at home. In addition, this HMD has two features to ensure safety. The first one is a restricted game zone to avoid blows, and on getting out, the real physical context 
will be displayed on the headset. Second, the virtual content of the session can be supervised through the Oculus app or via streaming, which is essential in telerehabilitation or home-based programmes. ${ }^{125}$

\section{Author affiliations}

${ }^{1}$ Department of Physiotherapy, University of Seville, Seville, Spain

${ }^{2}$ Dermatology Department, Virgen del Rocío University Hospital, Seville, Spain ${ }^{3}$ Department of Nursery, Physiotherapy and Occupational Therapy, University of Castilla-La Mancha, Toledo, Spain

${ }^{4}$ GIFT0, Physiotherapy Research Group, Toledo, Spain

\section{Twitter Elena Pinero-Pinto @elenapieropinto}

Contributors CGM, MDC-V and MJC-H conceptualised and designed the study. CGM wrote the first draft of the manuscript with critical input from MJC-H. MDC-V, MJC-H, JCH-R, EP-P and RPC contributed significantly to the revision of the manuscript. All authors read and approved the final manuscript.

Funding The authors have not declared a specific grant for this research from any funding agency in the public, commercial or not-for-profit sectors.

Competing interests None declared.

Patient consent for publication Not required.

Provenance and peer review Not commissioned; externally peer reviewed.

Supplemental material This content has been supplied by the author(s). It has not been vetted by BMJ Publishing Group Limited (BMJ) and may not have been peer-reviewed. Any opinions or recommendations discussed are solely those of the author(s) and are not endorsed by BMJ. BMJ disclaims all liability and responsibility arising from any reliance placed on the content. Where the content includes any translated material, BMJ does not warrant the accuracy and reliability of the translations (including but not limited to local regulations, clinical guidelines, terminology, drug names and drug dosages), and is not responsible for any error and/or omissions arising from translation and adaptation or otherwise.

Open access This is an open access article distributed in accordance with the Creative Commons Attribution Non Commercial (CC BY-NC 4.0) license, which permits others to distribute, remix, adapt, build upon this work non-commercially, and license their derivative works on different terms, provided the original work is properly cited, appropriate credit is given, any changes made indicated, and the use is non-commercial. See: http://creativecommons.org/licenses/by-nc/4.0/.

\section{ORCID iDs}

Cristina García-Muñoz http://orcid.org/0000-0003-2621-2098

María Jesús Casuso-Holgado http://orcid.org/0000-0002-4217-6827

Juan Carlos Hernández-Rodríguez http://orcid.org/0000-0003-2525-4069

Elena Pinero-Pinto http://orcid.org/0000-0001-9611-3939

Rocío Palomo-Carrión http://orcid.org/0000-0003-4034-2585

María-Dolores Cortés-Vega http://orcid.org/0000-0002-9514-8811

\section{REFERENCES}

1 Oh J, Vidal-Jordana A, Montalban X. Multiple sclerosis: clinical aspects. Curr Opin Neurol 2018;31:752-9.

2 Péran P, Nemmi F, Dutilleul C, et al. Neuroplasticity and brain reorganization associated with positive outcomes of multidisciplinary rehabilitation in progressive multiple sclerosis: a fMRI study. Mult Scler Relat Disord 2020;42:102127.

3 Ozgen G, Karapolat $\mathrm{H}$, Akkoc Y, et al. Is customized vestibular rehabilitation effective in patients with multiple sclerosis? A randomized controlled trial. Eur J Phys Rehabil Med 2016:52:466-78.

4 Van Emmerik REA, Remelius JG, Johnson MB, et al. Postural control in women with multiple sclerosis: effects of task, vision and symptomatic fatigue. Gait Posture 2010;32:608-14.

5 Barin L, Salmen A, Disanto G, et al. The disease burden of multiple sclerosis from the individual and population perspective: which symptoms matter most? Mult Scler Relat Disord 2018;25:112-21.

6 Gunn HJ, Newell P, Haas B, et al. Identification of risk factors for falls in multiple sclerosis: a systematic review and meta-analysis. Phys Ther 2013;93:504-13.

7 Dobson R, Giovannoni G. Multiple sclerosis - a review. Eur J Neurol 2019;26:27-40.
8 Manjaly Z-M, Harrison NA, Critchley HD, et al. Pathophysiological and cognitive mechanisms of fatigue in multiple sclerosis. $J$ Neurol Neurosurg Psychiatry 2019;90:642-51.

9 Hebert JR, Corboy JR, Manago MM, et al. Effects of vestibular rehabilitation on multiple sclerosis-related fatigue and upright postural control: a randomized controlled trial. Phys Ther 2011;91:1166-83.

10 Dhayalan D, Lund-Johansen M, Finnkirk M, et al. Fatigue in patients with vestibular schwannoma. Acta Neurochir 2019;161:1809-16.

11 Tramontano M, Martino Cinnera A, Manzari L, et al. Vestibular rehabilitation has positive effects on balance, fatigue and activities of daily living in highly disabled multiple sclerosis people: a preliminary randomized controlled trial. Restor Neurol Neurosci 2018;36:709-18.

12 Strupp M, Dlugaiczyk J, Ertl-Wagner BB, et al. Vestibular disorders. Dtsch Arztebl Int 2020;117:300-10.

13 Dieterich M. Central vestibular disorders. J Neurol 2007;254:559-68

14 Cochrane GD, Christy JB, Motl RW. Comprehensive clinical assessment of vestibular function in multiple sclerosis. $J$ Neurol Phys Ther 2021;45:228-34.

15 Klatt BN, Sparto PJ, Terhorst L, et al. Relationship between subjective visual vertical and balance in individuals with multiple sclerosis. Physiother Res Int 2019;24:1-7.

16 Doty RL, MacGillivray MR, Talab H, et al. Balance in multiple sclerosis: relationship to central brain regions. Exp Brain Res 2018;236:2739-50.

$17 \mathrm{Cao} \mathrm{H}$, Peyrodie L, Agnani O, et al. Evaluation of an expanded disability status scale (EDSS) modeling strategy in multiple sclerosis. Med Biol Eng Comput 2015;53:1141-51.

18 Nakamura J, Shiozaki T, Tsujimoto N, et al. Role of somatosensory and/or vestibular sensory information in subjective postural vertical in healthy adults. Neurosci Lett 2020;714:134598.

19 Hall CD, Heusel-Gillig L, Tusa RJ, et al. Efficacy of gaze stability exercises in older adults with dizziness. $J$ Neurol Phys Ther 2010;34:64-9.

20 Naranjo EN, Cleworth TW, Allum JHJ, et al. Vestibulo-spinal and vestibulo-ocular reflexes are modulated when standing with increased postural threat. J Neurophysiol 2016;115:833-42.

21 Tanaka H, Nakamura J, Siozaki T, et al. Posture influences on vestibulospinal tract excitability. Exp Brain Res 2021;239:997-1007.

22 Forbes PA, Siegmund GP, Schouten AC, et al. Task, muscle and frequency dependent vestibular control of posture. Front Integr Neurosci 2014:8:1-12.

23 Tusa RJ. Dizziness. Med Clin North Am 2009;93:263-71.

24 Murray AJ, Croce K, Belton T, et al. Balance control mediated by vestibular circuits directing limb extension or antagonist muscle coactivation. Cell Rep 2018;22:1325-38.

25 Zeigelboim BS, Arruda WO, Mangabeira-Albernaz PL, et al. Vestibular findings in relapsing, remitting multiple sclerosis: a study of thirty patients. Int Tinnitus J 2008;14:139-45.

26 Frohman EM, Kramer PD, Dewey RB, et al. Benign paroxysmal positioning vertigo in multiple sclerosis: diagnosis, pathophysiology and therapeutic techniques. Mult Scler 2003;9:250-5.

27 Di Stadio A, Ralli M, Altieri M, et al. Audiovestibular symptoms in patients with multiple sclerosis: a correlation between self-reported symptomatology and MRI findings to monitor disease progression. Mult Scler Relat Disord 2020;45:102431.

28 Dunlap PM, Holmberg JM, Whitney SL. Vestibular rehabilitation: advances in peripheral and central vestibular disorders. Curr Opin Neurol 2019;32:137-44.

29 Koura R, Hussein M. Vestibular-Evoked myogenic potential: an easy neurophysiological tool for evaluating brain stem involvement in multiple sclerosis. Egypt J Otolaryngol 2018;34:144-8.

30 Gabelić T, Krbot Skorić M, Adamec I, et al. The vestibular evoked myogenic potentials (VEMP) score: a promising tool for evaluation of brainstem involvement in multiple sclerosis. Eur J Neurol 2015;22:261-9.

31 Cooksey FS. Rehabilitation in vestibular injuries. Proc $R$ Soc Med 1946;39:273-8.

32 Tjernström F, Zur O, Jahn K. Current concepts and future approaches to vestibular rehabilitation. J Neurol 2016;263 Suppl 1:65-70.

33 Whitney SL, Alghwiri AA, Alghadir A. An overview of vestibular rehabilitation. 1st ed. Elsevier B.V, 2016.

34 Hebert JR, Corboy JR. The association between multiple sclerosisrelated fatigue and balance as a function of central sensory integration. Gait Posture 2013;38:37-42.

35 Hain TC. Neurophysiology of vestibular rehabilitation. NeuroRehabilitation 2011;29:127-41. 
36 Brown KE, Whitney SL, Marchetti GF, et al. Physical therapy for central vestibular dysfunction. Arch Phys Med Rehabil 2006;87:76-81

37 Han BI, Song HS, Kim JS. Vestibular rehabilitation therapy: review of indications, mechanisms, and key exercises. J Clin Neurol 2011;7:184-96.

38 García-Muñoz C, Cortés-Vega M-D, Heredia-Rizo AM, et al. Effectiveness of vestibular training for balance and dizziness rehabilitation in people with multiple sclerosis: a systematic review and meta-analysis. J Clin Med 2020;9:1-17.

39 Synnott E, Baker K. The effectiveness of vestibular rehabilitation on balance related impairments among multiple sclerosis patients: a systematic review. J Mult Scler 2020;7:1-8.

40 Meldrum D, Burrows L, Cakrt O, et al. Vestibular rehabilitation in Europe: a survey of clinical and research practice. J Neurol 2020;267:24-35.

41 Ricci NA, Aratani MC, Caovilla HH, et al. Evaluation of properties of the vestibular disorders activities of daily living scale (Brazilian version) in an elderly population. Braz J Phys Ther 2014;18:174-82.

42 Alahmari KA, Sparto PJ, Marchetti GF, et al. Comparison of virtual reality based therapy with customized vestibular physical therapy for the treatment of vestibular disorders. IEEE Trans Neural Syst Rehabil Eng 2014;22:389-99.

43 Yeh S-C, Chen S, Wang P-C, et al. Interactive 3-dimensional virtual reality rehabilitation for patients with chronic imbalance and vestibular dysfunction. Technol Health Care 2014;22:915-21.

44 Hsu S-Y, Fang T-Y, Yeh S-C, et al. Three-dimensional, virtual reality vestibular rehabilitation for chronic imbalance problem caused by Ménière's disease: a pilot study<sup/\&gt. Disabil Rehabil 2017;39:1601-6.

45 Micarelli A, Viziano A, Augimeri I, et al. Three-Dimensional headmounted gaming task procedure maximizes effects of vestibular rehabilitation in unilateral vestibular hypofunction: a randomized controlled pilot trial. Int J Rehabil Res 2017;40:325-32.

46 Micarelli A, Viziano A, Micarelli B, et al. Vestibular rehabilitation in older adults with and without mild cognitive impairment: effects of virtual reality using a head-mounted display. Arch Gerontol Geriatr 2019;83:246-56.

47 Meldrum D, Herdman S, Vance R, et al. Effectiveness of conventional versus virtual reality-based balance exercises in vestibular rehabilitation for unilateral peripheral vestibular loss: results of a randomized controlled trial. Arch Phys Med Rehabil 2015;96:1319-28.

48 Viziano A, Micarelli A, Augimeri I, et al. Long-Term effects of vestibular rehabilitation and head-mounted gaming task procedure in unilateral vestibular hypofunction: a 12-month follow-up of a randomized controlled trial. Clin Rehabil 2019;33:24-33.

49 Yeh S-C, Huang M-C, Wang P-C, et al. Machine learning-based assessment tool for imbalance and vestibular dysfunction with virtual reality rehabilitation system. Comput Methods Programs Biomed 2014;116:311-8.

50 Rosiak O, Krajewski K, Woszczak M, et al. Evaluation of the effectiveness of a virtual Reality-based exercise program for unilateral peripheral vestibular deficit. VES 2019;28:409-15.

51 Casuso-Holgado MJ, Martín-Valero R, Carazo AF, et al. Effectiveness of virtual reality training for balance and gait rehabilitation in people with multiple sclerosis: a systematic review and meta-analysis. Clin Rehabil 2018;32:1220-34.

52 Xie M, Zhou K, Patro N, et al. Virtual reality for vestibular rehabilitation: a systematic review. Otol Neurotol 2021;42:967-77.

53 Yamagami M, Imsdahl S, Lindgren K, et al. Effects of virtual reality environments on overground walking in people with Parkinson disease and freezing of gait. Disabil Rehabil Assist Technol 2020;0:1-8

54 Weiss P, Keshner E, Levin M. Virtual reality for physical and motor rehabilitation. Springer International Publishing, 2014.

$55 \mathrm{Kim} \mathrm{J-H}$. Effects of a virtual reality video game exercise program on upper extremity function and daily living activities in stroke patients. $J$ Phys Ther Sci 2018;30:1408-11.

56 Lee H-S, Lim J-H, Jeon B-H, et al. Non-immersive virtual reality rehabilitation applied to a Task-oriented approach for stroke patients: a randomized controlled trial. Restor Neurol Neurosci 2020;38:165-72.

57 Kramer A, Dettmers C, Gruber M. Exergaming with additional postural demands improves balance and gait in patients with multiple sclerosis as much as conventional balance training and leads to high adherence to home-based balance training. Arch Phys Med Rehabil 2014;95:1803-9.

58 Gatica-Rojas V, Méndez-Rebolledo G. Virtual reality interface devices in the reorganization of neural networks in the brain of patients with neurological diseases. Neural Regen Res 2014:9:888-96.

59 Mat Rosly M, Mat Rosly H, Davis Oam GM, et al. Exergaming for individuals with neurological disability: a systematic review. Disabil Rehabil 2017;39:727-35.

60 Robinson J, Dixon J, Macsween A, et al. The effects of exergaming on balance, gait, technology acceptance and flow experience in people with multiple sclerosis: a randomized controlled trial. BMC Sports Sci Med Rehabil 2015;7:8.

61 Glen K, Eston R, Loetscher T, et al. Exergaming: feels good despite working harder. PLoS One 2017;12:e0186526.

62 Whitney SL, Sparto PJ. Principles of vestibular physical therapy rehabilitation. NeuroRehabilitation 2011;29:157-66.

63 Szpak A, Michalski SC, Loetscher T. Exergaming with beat Saber: an investigation of virtual reality aftereffects. J Med Internet Res 2020;22:e19840.

64 Servotte J-C, Goosse M, Campbell SH, et al. Virtual reality experience: immersion, sense of presence, and Cybersickness. Clinical Simulation in Nursing 2020;38:35-43

65 Chan A-W, Tetzlaff JM, Altman DG, et al. Spirit 2013 statement: defining standard protocol items for clinical trials. Ann Intern Med 2013;158:200-7.

66 Schulz KF, Altman DG, Moher D, et al. Consort 2010 statement: updated guidelines for reporting parallel group randomised trials. BMJ 2010;340:c332.

67 Le M, Malpas C, Sharmin S, et al. Disability outcomes of early cerebellar and brainstem symptoms in multiple sclerosis. Mult Scler 2021;27:755-66.

68 Flachenecker P, Kümpfel T, Kallmann B, et al. Fatigue in multiple sclerosis: a comparison of different rating scales and correlation to clinical parameters. Mult Scler 2002;8:523-6.

69 Viveiro LAP, Gomes GCV, Bacha JMR, et al. Reliability, validity, and ability to identity fall status of the Berg balance scale, balance evaluation systems test (BESTest), Mini-BESTest, and Brief-BESTest in older adults who live in nursing homes. J Geriatr Phys Ther 2019;42:E45-54.

70 Kalincik T. Multiple sclerosis relapses: epidemiology, outcomes and management. A systematic review. Neuroepidemiology 2015;44:199-214.

71 Burina A, Sinanović O, Smajlović D, et al. Some aspects of balance disorder in patients with multiple sclerosis. Bosn J Basic Med Sci 2008;8:80-5.

72 Noble H, Heale R. Triangulation in research, with examples. Evid Based Nurs 2019;22:67-8.

73 Hall CD, Cox LC. The role of vestibular rehabilitation in the balance disorder patient. Otolaryngol Clin North Am 2009;42:161-9.

74 Afrasiabifar A, Karami F, Najafi Doulatabad S. Comparing the effect of Cawthorne-Cooksey and Frenkel exercises on balance in patients with multiple sclerosis: a randomized controlled trial. Clin Rehabil 2018;32:57-65.

75 Karami F, Afrasiabifar A, Najafi Doulatabad S. Comparing the effectiveness of vestibular rehabilitation and frenkel exercise on fatigue reduction in patients with multiple sclerosis: a randomized controlled trial. Iran Red Crescent Med J 2018; In Press.

76 Lai B, Davis D, Narasaki-Jara M, et al. Feasibility of a commercially available virtual reality system to achieve exercise guidelines in youth with spina bifida: mixed methods case study. JMIR Serious Games 2020;8:e20667

77 Saker M, Frith J. Coextensive space: virtual reality and the developing relationship between the body, the digital and physical space. Media, Culture \& Society 2020;42:1427-42.

78 Newberry A, Sherwood P, Hricik A, et al. Understanding recruitment and retention in neurological research. J Neurosci Nurs 2010;42:47-57.

79 Bell ML, Kenward MG, Fairclough DL, et al. Differential dropout and bias in randomised controlled trials: when it matters and when it may not. BMJ 2013;346:e8668.

80 Brooke J. Sus: a retrospective. JUS 2013;8:29-40.

81 Bangor A, Kortum PT, Miller JT. An empirical evaluation of the system usability scale. Int J Hum Comput Interact 2008;24:574-94

82 Saredakis D, Szpak A, Birckhead B, et al. Factors associated with virtual reality sickness in head-mounted displays: a systematic review and meta-analysis. Front Hum Neurosci 2020;14:96.

83 Micklewright D, St Clair Gibson A, Gladwell V, et al. Development and validity of the Rating-of-Fatigue scale. Sports Med 2017;47:2375-93.

84 Lubetzky AV, Kelly J, Wang Z, et al. Contextual sensory integration training via head mounted display for individuals with vestibular disorders: a feasibility study. Disabil Rehabil Assist Technol 2020:1-11. 
85 Ventura S, Brivio E, Riva G, et al. Immersive versus Non-immersive experience: exploring the feasibility of memory assessment through $360^{\circ}$ technology. Front Psychol 2019;10:2509.

86 Van De Wyngaerde KM, Lee MK, Jacobson GP, et al. The component structure of the dizziness handicap inventory (DHI): a reappraisal. Otol Neurotol 2019;40:1217-23.

87 Tamber A-L, Wilhelmsen KT, Strand LI. Measurement properties of the dizziness handicap inventory by cross-sectional and longitudinal designs. Health Qual Life Outcomes 2009;7:101.

88 Yorke A, Ward I, Vora S, et al. Measurement characteristics and clinical utility of the dizziness handicap inventory among individuals with vestibular disorders. Arch Phys Med Rehabil 2013;94:2313-4.

89 Cattaneo D, Jonsdottir J, Repetti S. Reliability of four scales on balance disorders in persons with multiple sclerosis. Disabil Rehabil 2007;29:1920-5.

90 Cattaneo D, Regola A, Meotti M. Validity of six balance disorders scales in persons with multiple sclerosis. Disabil Rehabil 2006;28:789-95.

91 Cachupe WJC, Shifflett B. Measurement in physical education and exercise science reliability of Biodex balance system measures 2009:37-41.

92 Parraca JA, Olivares PR, Carbonell-Baeza A, et al. Test-Retest reliability of Biodex balance SD on physically active old people. JHSE 2011;6:444-51.

93 Drouin JM, Valovich-mcLeod TC, Shultz SJ, et al. Reliability and validity of the Biodex system 3 pro isokinetic dynamometer velocity, torque and position measurements. Eur J Appl Physiol 2004;91:22-9.

94 Ghait AS, Elheneidi El, Shendy WS, et al. Evaluation of stability and postural control in patients with multiple sclerosis pre and post balance program on Biodex balance system. IJPR 2019;7:2993-6.

95 Atteya A, Elwishy A, Kishk N, et al. Assessment of postural balance in multiple sclerosis patients. Egypt $J$ Neurol Psychiatry Neurosurg 2019;55

96 Mehta T, Young H-J, Lai B, et al. Comparing the convergent and concurrent validity of the dynamic gait index with the Berg balance scale in people with multiple sclerosis. Healthcare 2019;7:27.

97 Moore JL, Potter K, Blankshain K, et al. A core set of outcome measures for adults with neurologic conditions undergoing rehabilitation: a clinical practice guideline. J Neurol Phys Ther 2018;42:174-220.

98 Gervasoni E, Jonsdottir J, Montesano A, et al. Minimal clinically important difference of Berg balance scale in people with multiple sclerosis. Arch Phys Med Rehabil 2017;98:337-40.

99 Marchesi O, Vizzino C, Meani A, et al. Fatigue in multiple sclerosis patients with different clinical phenotypes: a clinical and magnetic resonance imaging study. Eur J Neurol 2020;27:2549-60.

100 Taul-Madsen L, Dalgas U, Kjølhede T, et al. A head-to-head comparison of an isometric and a concentric fatigability protocol and the association with fatigue and walking in persons with multiple sclerosis. Neurorehabil Neural Repair 2020;34:523-32.

101 Larson RD. Psychometric properties of the modified fatigue impact scale. Int J MS Care 2013;15:15-20.

102 Mathiowetz V. Test-Retest reliability and convergent validity of the fatigue impact scale for persons with multiple sclerosis. $A m \mathrm{~J}$ Occup Ther 2003;57:389-95.

103 Rietberg MB, Van Wegen EEH, Kwakkel G. Measuring fatigue in patients with multiple sclerosis: reproducibility, responsiveness and concurrent validity of three Dutch self-report questionnaires. Disabil Rehabil 2010;32:1870-6.

104 Rooney S, McFadyen DA, Wood DL, et al. Minimally important difference of the fatigue severity scale and modified fatigue impact scale in people with multiple sclerosis. Mult Scler Relat Disord 2019;35:158-63.

105 Heiskanen S, Meriläinen P, Pietilä A-M. Health-Related quality of life-testing the reliability of the MSQOL-54 instrument among MS patients. Scand J Caring Sci 2007;21:199-206.

106 Ochoa-Morales A, Hernández-Mojica T, Paz-Rodríguez F, et al. Quality of life in patients with multiple sclerosis and its association with depressive symptoms and physical disability. Mult Scler Relat Disord 2019;36:101386.
107 Ricci NA, Aratani MC, Caovilla HH, et al. Effects of vestibular rehabilitation on balance control in older people with chronic dizziness: a randomized clinical trial. Am J Phys Med Rehabil 2016:95:256-69.

108 Lavalle SM, Yershova A, Katsev M. Head tracking for the oculus Rift. Proc - IEEE Int Conf Robot Autom 2014:187-94.

109 Lubetzky AV, Wang Z, Krasovsky T. Head mounted displays for capturing head kinematics in postural tasks. J Biomech 2019;86:175-82.

$110 \mathrm{Xu}$ X, Chen KB, Lin J-H, et al. The accuracy of the oculus Rift virtual reality head-mounted display during cervical spine mobility measurement. J Biomech 2015:48:721-4.

111 Rosiak O, Jozefowicz-Korczynska M. Role of head-mounted displays in enhancing vestibular rehabilitation effects: Comment on "Evaluation of the effectiveness of a Virtual Reality-based exercise program for Unilateral Peripheral Vestibular Deficit". VES 2019:1-2.

112 Kourtesis P, Collina S, Doumas LAA, et al. Technological competence is a Pre-condition for effective implementation of virtual reality head mounted displays in human neuroscience: a technological review and meta-analysis. Front Hum Neurosci 2019;13:1-17.

113 Stanney K, Lawson BD, Rokers B, et al. Identifying causes of and solutions for Cybersickness in immersive technology: reformulation of a research and development agenda. Int J Hum Comput Interact 2020;36:1783-803.

114 Weech S, Kenny S, Barnett-Cowan M. Presence and Cybersickness in virtual reality are negatively related: a review. Front Psychol 2019;10:158.

115 AbouShady N, Kamel A, Ibrahim R. Cawthorne Cooksey versus habituation training in Vertebro basilar insufficiency patients. International Journal of Therapies and Rehabilitation Research 2017;6:91.

116 Kulcu DG, Yanik B, Boynukalin S, et al. Efficacy of a homebased exercise program on benign paroxysmal positional vertigo compared with betahistine. J Otolaryngol Head Neck Surg 2008;37:373-9.

117 Gottshall KR, Sessoms PH. Improvements in dizziness and imbalance results from using a multi disciplinary and multi sensory approach to vestibular physical therapy - a case study. Front Syst Neurosci 2015;9:1-7.

118 Soltani P, Andrade R. The influence of virtual reality head-mounted displays on balance outcomes and training paradigms: a systematic review. Front Sports Act Living 2020;2:531535.

119 Meldrum D, Herdman S, Moloney R, et al. Effectiveness of conventional versus virtual reality based vestibular rehabilitation in the treatment of dizziness, gait and balance impairment in adults with unilateral peripheral vestibular loss: a randomised controlled trial. BMC Ear Nose Throat Disord 2012;12:3.

120 Meldrum D, Glennon A, Herdman S, et al. Virtual reality rehabilitation of balance: assessment of the usability of the Nintendo Wii(囚) Fit Plus. Disabil Rehabil Assist Technol 2012;7:205-10.

121 Massetti T, da Silva TD, Crocetta TB, et al. The clinical utility of virtual reality in neurorehabilitation: a systematic review. J Cent Nerv Syst Dis 2018;10:117957351881354.

122 Peruzzi A, Cereatti A, Della Croce U, et al. Effects of a virtual reality and treadmill training on gait of subjects with multiple sclerosis: a pilot study. Mult Scler Relat Disord 2016;5:91-6.

123 Gutiérrez RO, Galán Del Río F, Cano de la Cuerda R, et al. A telerehabilitation program by virtual reality-video games improves balance and postural control in multiple sclerosis patients. NeuroRehabilitation 2013;33:545-54.

124 Chanpimol S, Benson K, Maloni H, et al. Acceptability and outcomes of an individualized exergaming telePT program for veterans with multiple sclerosis: a pilot study. Arch Physiother 2020;10:1-10.

125 Morone G, Girardi S, Ghanbari Ghooshchy S. Wearable devices and virtual reality for neurorehabilitation: an opportunity for home rehabilitation. Springer International Publishing, 2019. 